\title{
Laser processing effect on magnetic properties of amorphous wires
}

Cite as: Appl. Phys. Lett. 81, 4790 (2002); https://doi.org/10.1063/1.1528724

Submitted: 18 June 2002 . Accepted: 21 October 2002 . Published Online: 09 December 2002

A. N. Grigorenko, L. V. Panina, S. I. Sandacci, D. P. Makhnovskiy, D. J. Mapps, I. Y. Khrushchev, M. V. Dubov, V. Larin, and A. Torkunov

\section{ARTICLES YOU MAY BE INTERESTED IN}

Stress effect on magnetoimpedance in amorphous wires at gigahertz frequencies and application to stress-tunable microwave composite materials

Journal of Applied Physics 97, 013701 (2005); https://doi.org/10.1063/1.1821640

\section{Applied Physics Reviews} Now accepting original research 


\title{
Laser processing effect on magnetic properties of amorphous wires
}

\author{
A. N. Grigorenko, ${ }^{\text {a) }}$ L. V. Panina, S. I. Sandacci, D. P. Makhnovskiy, and D. J. Mapps \\ Department of Communications and Electrical Engineering, University of Plymouth, Drake Circus, \\ Plymouth PL4 8AA, United Kingdom \\ I. Y. Khrushchev and M. V. Dubov \\ Department of Electronic Engineering, Aston University, Aston Triangle, Birmingham B4 7ET, \\ United Kingdom \\ V. Larin and A. Torkunov \\ Amotec Ltd., Kishinev, Moldova
}

(Received 18 June 2002; accepted 21 October 2002)

\begin{abstract}
Controllable, spatially selective treatment of glass-covered amorphous magnetic wires by laser irradiation is demonstrated. Local annealing by an argon laser has resulted in the formation of weakly interacting heterogeneous structures with different magnetic properties. Two large and stable Barkhausen jumps were detected in the magnetization reversal of high and positive magnetostriction wires with treated portions of $200 \mu \mathrm{m}$ and $0.5 \mathrm{~cm}$. The local annealing of negative magnetostrictive wires has modified wire magnetoimpedance: the maximum of the impedance plot flattens and the working field interval increases. These favorable changes are due to the creation of local stresses and structure interfaces. (C) 2002 American Institute of Physics. [DOI: 10.1063/1.1528724]
\end{abstract}

Amorphous thin wires are among the most interesting materials for applications as magnetic sensing elements, owing to their unique properties related to reentrant magnetic flux reversal ${ }^{1-3}$ and giant magneto impedance. ${ }^{4,5}$ These properties are influenced by domain structures that exist in the as-cast state and can be modified by various annealing methods. ${ }^{6-8}$ Amorphous materials do not possess magnetocrystalline anisotropy. Therefore, the anisotropy and the domain structure are determined by the balance between the magnetoelastic and magnetostatic (shape) contributions. The alloy composition determines the value and sign of the magnetostrictive constant whereas the fabrication process and annealing under stress and/or magnetic field results in a particular magnetoelastic energy. Then, various kinds of magnetic structures can be realized by a suitable postproduction treatment, which normally affects the whole sample. A local processing, which influences only a part of an amorphous material, is also possible. For example, laser radiation was used to achieve local crystallization of amorphous CoP alloys, ${ }^{9}$ to change locally the anisotropy of ferromagnetic amorphous ribbons, ${ }^{10}$ and to cause local phase transitions to the ferromagnetic state in thin amorphous nonmagnetic $\mathrm{CoC}$ films. ${ }^{11}$ However, little work has been done to investigate the effect of a local treatment of amorphous materials with the aim to produce heterogeneous magnetic structures. In particular, the development of a method for the predetermined, spatially patterned modification of glass covered amorphous wires would be a very attractive proposition.

In this letter we present our results on local annealing of glass-covered amorphous magnetic wires by laser light. We demonstrate that magnetic properties of amorphous wires can be locally and controllably tuned by argon laser processing due to the formation of magnetic heterostructures. This can be of great interest for application of amorphous wires as magnetic labels and tags.

The sketch of the installation is shown in Fig. 1. In the experiment, the magnetic wires were exposed to the emission of an argon laser operating at a wavelength of $488 \mathrm{~nm}$. The laser beam was focused into an elliptical spot, aligned with the wire, by a positive cylindrical lens. The width of the spot across the wire was about of $25 \mu \mathrm{m}$. An additional negative cylindrical lens was used for expanding the laser spot along the wire. Depending on the position of the negative lens, the spot length varied from $\sim 2$ to $10 \mathrm{~mm}$. It was possible to focus the beam tighter, down to a length of $30 \mu \mathrm{m}$, by substituting the negative lens with a microscope objective.

A glass-covered amorphous wire was fixed on a bracket holder that allowed an exposure of the middle section of the wire. A three-coordinate translation stage provided precise alignment of the holder. The maximal power in the beam was $6.5 \mathrm{~W}$, corresponding to the maximum intensity in the focus in excess of $10^{4} \mathrm{~W} / \mathrm{cm}^{2}$.

We used wires of the following compositions: $\mathrm{Fe}_{74} \mathrm{~B}_{13} \mathrm{Si}_{11} \mathrm{C}_{2}$ (sample 1), $\mathrm{Fe}_{36} \mathrm{Co}_{40} \mathrm{~B}_{13} \mathrm{Si}_{11}$ (sample 2), and $\mathrm{Co}_{68} \mathrm{Fe}_{4} \mathrm{~B}_{11} \mathrm{Si}_{14}(\mathrm{NiMoGe})_{3}$ (sample 3 ). Depending on $\mathrm{Co} / \mathrm{Fe}$ content, these materials have large positive magnetostriction

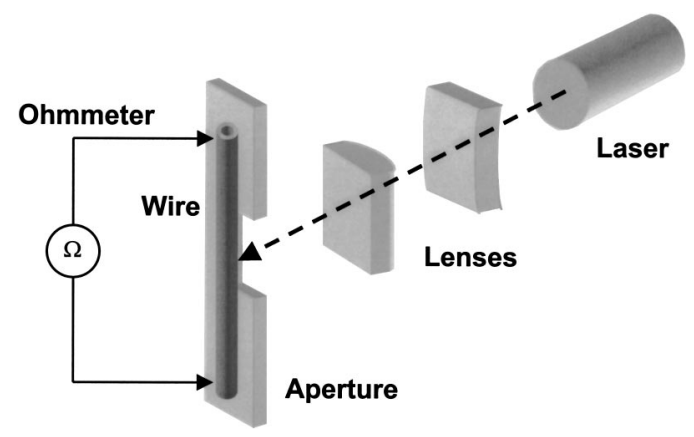

FIG. 1. Schematic of installation.

${ }^{a)}$ Electronic mail: a.grigorenko@plymouth.ac.uk 
(samples 1 and 2) or small negative magnetostriction (sample 3), which results in different magnetic anisotropies. The general magnetic properties and the fabrication method of glass-covered amorphous wires are summarized in Refs. 12 and 13. An axial anisotropy and a longitudinal domain structure are typical of positive magnetostrictive wires, whereas a circumferential anisotropy and circular domain structure exist in the outer region of negative magnetostrictive wires. In the first case, the longitudinal magnetization is characterized by a large Barkhausen jump leading to bistable rectangular magnetization loops. In the second case, the wire impedance can be sensitively controlled by a small axial magnetic field owing to the circular magnetization process. Both types of wires have been laser processed to investigate the effect of this treatment on the bistability and giant magnetoimpedance (GMI).

In the experiment, we exposed both types of wires to the laser light and investigated the changes in the bistable behavior of samples 1 and 2 as well as the giant magnetoimpedance (GMI) in sample 3 before and after the treatment.

A change in the wire resistance was used as a reference parameter for the laser treatment. The dependence of the wire resistance upon temperature has been calibrated in a thermal bath prior to processing. The wire resistance changed with temperature at a rate $\eta=\Delta R /(R \cdot \Delta T)=1.2$ $\times 10^{-4} \mathrm{C}^{-1}$. Therefore, knowing the length of the exposed section, we can roughly evaluate the local temperature of the treated wire from the total resistance of the sample. When a wire was exposed to relatively low laser intensity below $10^{3} \mathrm{~W} / \mathrm{cm}^{2}$, its resistance changed reversibly, i.e., the resistance increased and returned to the initial value after irradiation. At a higher level of exposure, the wire resistance dropped irreversibly by $1 \%-3 \%$. The likely reason for that is crystallization of the sample and a better conductivity of a crystalline state. During the experiments, care was taken to avoid the crystallization process. The annealing procedure consisted of an increase and a decrease of laser power until the total wire resistance fell to a predetermined value below the initial value. The exposure time was typically of a minute.

In the wires with positive magnetostriction (samples 1 and 2), we investigated the effect of laser treatment on the magnetic reversal characteristics. The magnetization curves were measured by a conventional inductive method using two differential pickup coils of 30 turns, $0.5 \mathrm{~mm}$ in inner diameter $(0.8 \mathrm{~mm}$ in outer diameter $)$ and $1 \mathrm{~cm}$ long. Figures 2(a) and 2(c) show the measured differential response of an unprocessed wire and that of a partially laser treated wire (sample 1), respectively. The total sample length was $2 \mathrm{~cm}$ and the length of the treated portion was about $5 \mathrm{~mm}$. For as-cast wires, a sharp voltage pulse was detected at a magnetic field of 0.7 Oe [see Fig. 2(a)]. This pulse is characteristic of a large Barkhausen jump. Placing the processed wire in such a way that the detection coil contained both treated and nontreated portions, we detected two voltage pulses (two large Barkhausen jumps) shown in Fig. 2(c). The first pulse corresponds to that of Fig. 2(a) and the other occurs at higher field of 1.9 Oe. On the other hand, if the treated portion was outside the detection coil there was no change in the magnetization reversal behavior in comparison with as-cast wires
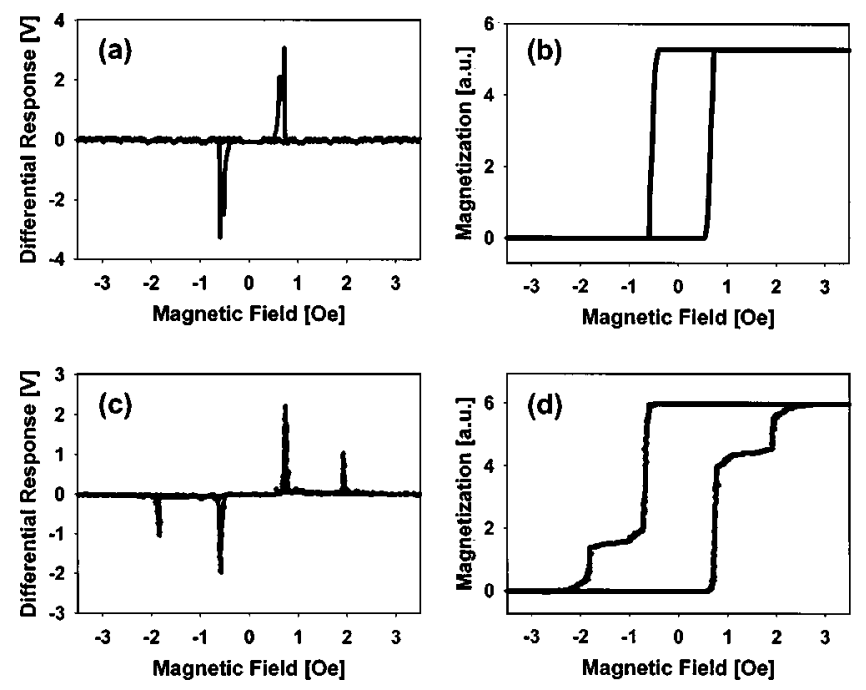

FIG. 2. Magnetic responses of a laser treated bistable amorphous wiresample 1. The differential signals and reconstructed magnetization loops of the as-cast wire (a) and (b) and the processed wire (c) and (d). The laser treated portion is about $0.5 \mathrm{~cm}$, evaluated annealing temperature is about $500{ }^{\circ} \mathrm{C}$.

[Fig. 2(a)]. Therefore, the laser annealing induced a local change in coercivity and produced no measurable effect on the adjacent areas. By reducing the width of the pickup coils and measuring local magnetization loops, we confirmed that the magnetization reversal happens in treated and nontreated portions by means of a large Barkhausen jump occurring at different fields. As a result, the differential response shown in Fig. 2(c) has two pulses corresponding to the coercivity of processed and unprocessed material. Similarly, one can compare the reconstructed hysteresis loops in Fig. 2(b) (unprocessed wire) and Fig. 2(d) (partially annealed sample 1). The hysteresis loop of sample 1 consists of a superposition of two rectangular hysteresis loops with two distinctly different coercivities. It means that a locally laser treated bistable wire represents an interesting prototype of a simple 2-bit element for product labeling.

Next we studied the effect of the length of the annealed portion on the appearance of the second magnetization jump. Using the resistance change to control annealing, we can reliably monitor the resistance changes (and hence temperature) of a wire with a treated portion down to about $100 \mu \mathrm{m}$. Even in this case, two voltage pulses appear at the magnetization reversal, which is demonstrated in Fig. 3(a) for sample 2 with the annealed part of $200 \mu \mathrm{m}$. The treated area was reduced further by making use of spherical optics. The resistance control was unreliable for small dimensions of the treated part because the wire resistance changes were small.

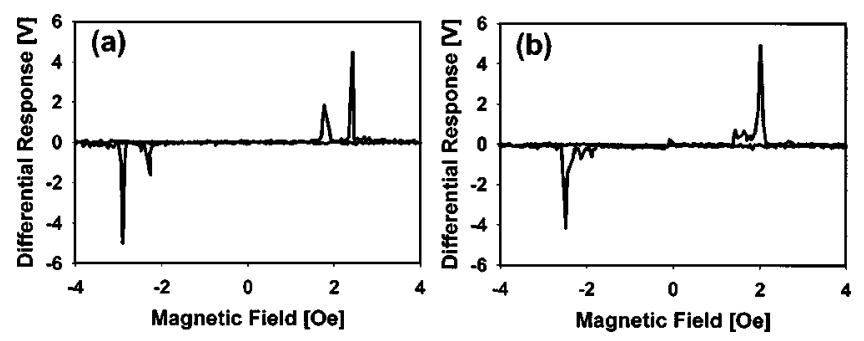

FIG. 3. Magnetic responses of a laser treated bistable amorphous wiresample 2. The differential signals of the processed wire: (a) the annealing length of $200 \mu \mathrm{m}$, (b) the annealing length $30 \mu \mathrm{m}$. 

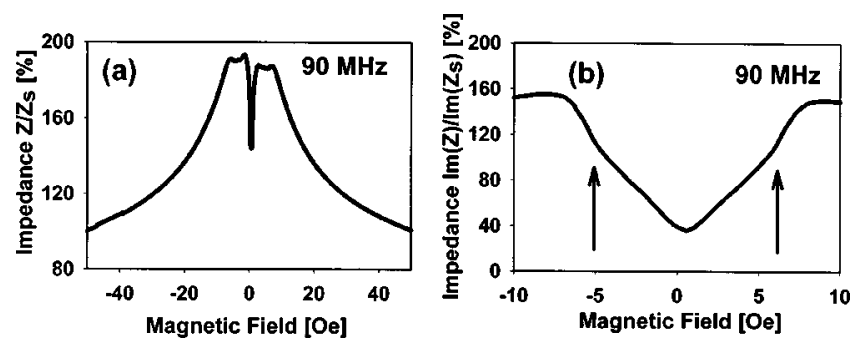

FIG. 4. Magnetoimpedance characteristic of a laser annealed GMI wire at frequency $90 \mathrm{MHz}$-sample 3: (a) impedance dependence upon magnetic field, (b) the imaginary part of impedance as a function of small fields. Impedance is normalized by the saturation impedance value $Z_{s}$. The laser treated portion is about $0.5 \mathrm{~cm}$, evaluated annealing temperature is about $500{ }^{\circ} \mathrm{C}$.

Instead, an intensity of the laser beam was taken as an indicator of the annealing temperature. Figure 3(b) demonstrates that there is no second large Barkhausen jump at the magnetization process after annealing of a $30 \mu \mathrm{m}$ portion of sample 2. This gives $\sim 100 \mu \mathrm{m}$ as the upper limit of the wire laser treated length at which distinct magnetic properties are developed and the second magnetization jump is achieved.

It is interesting to note that the amplitude of the pulse related to the annealed portion [seen in Fig. 3(a) at smaller fields and in Fig. 2(b) at higher fields] is relatively large despite a small contribution of this area to the total interrogated region. This observation is very useful for wire applications as a label. It is explained by the fact that the reversal domains have an elongated shape ${ }^{14}$ and local laser annealing creates interfaces which act as pinning or nucleation sites for those domains. Also, the measured pulse time is proportional to the length of the "reversing" portion of the wire inside the pickup coil, which makes peaks of Fig. 2(c) narrower than those of Fig. 2(a).

In the case of amorphous wires with a circular domain structure (sample 3) the laser treatment also forms a heterogenic magnetic system, which can be seen from GMI characteristics. Figure 4(a) plots the relative change in impedance as a function of axial magnetic field for a processed wire with the total length of $1 \mathrm{~cm}$ and treated portion of about 5 $\mathrm{mm}$. A notable feature of this dependence is the existence of a flat insensitive region in the field range from 2 to 7 Oe. The plateau comes from the superposition of the GMI characteristics from the laser annealed and unprocessed parts of the wire, which peak at different values of the external field. Figure 4(b) shows the plot of the imaginary part of the wire impedance for low fields, where two regions with different GMI slopes and field ranges are clearly seen. The up and down magnetoimpedance curves shown in Fig. 4 coincide. This implies that the treated part of the wire changed the magnitude but conserved the circumferential nature of anisotropy. Such GMI characteristics can be useful for applications in magnetic proximity and switching sensors.

We briefly discuss a possible mechanism of the observed effect. The favorable changes of the magnetic properties cannot be attributed to the partial crystallization of the wires. When crystallization took place and the wire resistance dropped considerably after processing, we found that magnetization reversal of magnetic wires with positive magneto- striction did not happen in one large Barkhausen jump and hysteresis loops lost their rectangular shape. This is due to the fact that crystallization usually results in multiple pinning centers instead of homogeneous local changes. The local change in the magnetic properties is most likely connected with the stress induced by the glass cover in the metallic core. During irradiation the laser power is absorbed mainly inside the core. The core expands and heats up the surrounding glass. As a result, the mechanical equilibrium and local stress of the glass-covered wires are changed when radiation is removed and sample is cooled to an ambient temperature. Scanning electron and optical microscopy of laser-annealed wires revealed that the glass cover changed its form in the laser treated parts of the wire and there is a tendency of developing of microgaps between the glass and the core. This may induce nucleation centers in the amorphous material and influence its magnetic characteristics. It is worth noting similar magnitudes of the switching fields for treated portions obtained for samples 1 and 2, which may suggest that the energy barriers associated with the produced nucleation centers are mostly determined by the annealing characteristics. Further investigation is required to clarify the mechanism of laser annealing.

In conclusion, we applied laser radiation for local, reliable, and controllable treatment of different glass-covered amorphous wires. We have shown that the formation of heterogeneous magnetic structures with uniform properties can be realized by this method. The magnetic properties of these materials such as a large Barkhausen jump and giant magnetoimpedance can be locally adjusted in order to match a particular application. The proposed technique is suitable for mask or multistage laser microprocessing and can be used for fabrication of micro- (nano-) structures based on amorphous magnetic materials.

This work was supported in part by EPSRC Grant Nos. GR/N02283/01 and GR/M23977/01.

${ }^{1}$ R. C. O’Handley, J. Appl. Phys. 46, 4996 (1975).

${ }^{2}$ F. B. Humphrey, K. Mohri, J. Yamasaki, K. Kawamura, R. Malmhall, and I. Ogasawara, Magnetic Properties of Amorphous Metals, New York, (1987).

${ }^{3}$ H. Chiriac, T. A. Ovari, M. Vazquez, and A. Hernando, J. Magn. Magn. Mater. 177, 205 (1998).

${ }^{4}$ L. V. Panina and K. Mohri, Appl. Phys. Lett. 65, 1189 (1994).

${ }^{5}$ M. Vazquez, J. Magn. Magn. Mater. 226, 693 (2001).

${ }^{6}$ R. Malmhal, K. Mohri, F. B. Humphrey, T. Manabe, H. Kawamura, J. Yamasaki, and I. Ogasawara, IEEE Trans. Magn. 23, 3242 (1987).

${ }^{7}$ J. Gonzalez, N. Murillo, V. Larin, J. M. Barandiaran, M. Vazquez, and A. Hernando, Sens. Actuators A 59, 97 (1997).

${ }^{8}$ V. Zhukova, A. F. Cobeno, A. Zhukov, J. M. Blanco, S. Puerta, J. Gonzalez, and M. Vazquez, J. Non-Cryst. Solids 287, 31 (2001).

${ }^{9}$ M. Zheng, M. Yu, Y. Liu, R. Skomski, S. H. Liou, D. J. Sellmyer, V. N. Petryakov, Y. K. Verevkin, N. I. Polushkin, and N. N Salashchenko, Appl. Phys. Lett. 79, 2606 (2001).

${ }^{10}$ M. Garcia and M. C. Sanchez, Rev. Phys. Appl. 21, 207 (1986).

${ }^{11}$ C. Aroca, M. C. Sanchez, I. Tanarro, P. Sanchez, E. Lopez, and M. Vazquez, Phys. Rev. B 42, 8086 (1990).

${ }^{12}$ H. Chiriac, Mater. Sci. Eng., A 304, 166 (2001).

${ }^{13}$ A. Zhukov, J. Gonzalez, J. M. Blanco, M. Vazquez, and V. Larin, J. Mater. Res. 15, 2107 (2000).

${ }^{14}$ L. V. Panina, H. Katoh, M. Mizutani, K. Mohri, and F. B. Humphrey, IEEE Trans. Magn. 28, 2922 (1992). 